\title{
Linking fat intake, the intestinal microbiome, and necrotizing enterocolitis in premature infants
}

\author{
Daniel T. Robinson ${ }^{1}$ and Michael S. Caplan ${ }^{2}$
}

Components of diet, including the total amounts and specific types of fat, affect the composition of the intestinal microbiome in both animal models and cohort studies of humans. Amounts of total fat and specific fatty acids (FA) are some of the most variable nutritional components of breast milk. Evaluations of the microbiome in premature infants have shown decreased diversity of species and increased proportions of potentially pathogenic bacteria. Microbial patterns in premature infants may be affected by nutritional fat intake, altering risk of diseases such as necrotizing enterocolitis. Dietary FA may also impact disease susceptibility through molecular mechanisms. Specifically, intestinal Toll-like receptor 4 expression is altered by manipulation of FA in murine models. Abnormal increased expression of Toll-like receptor 4, the receptor for lipopolysaccharide, has been implicated in necrotizing enterocolitis. This report will review the role of dietary fat in the composition of the intestinal microbiome, the extreme variability of FA intake in premature infants, and associations of both dysbiosis and FA intake with the development of necrotizing enterocolitis.

W ith plausible relationships between the intestinal microbiome and disease risk, scientists continue to explore factors that affect diversity and flux of the intestinal flora in neonates and infants. Intestinal bacteria metabolize macronutrients to create essential products such as vitamins and energy sources and yet macronutrients may influence the composition and functions of the microbiome. Fat intake contributes over $40 \%$ of the total energy (TE) ingested by infants, often the highest proportion of energy, and thus this nutrient deserves attention as a possible influential factor in microbial composition.

Prematurity-related influences affect intestinal colonization including higher rates of cesarean delivery, antibiotic exposure, delayed exposure to breast milk feedings, and significant delays in breastfeeding due to immaturity (1-3). Comparison findings between premature and term neonates include interindividual diversity in both groups and reduced species diversity in those born premature $(3,4)$. Feeding breast milk vs. formula may lead to different grouping patterns and variability (2).

This review will discuss the influences of dietary fat intake on the microbiome as documented in animal and human studies, the variability of fat intake and absorption by premature infants, as well as the relationship of fatty acids (FA), microbes and Toll-like receptor 4 (TLR4) activation with necrotizing enterocolitis (NEC).

\section{CONTROLLED ALTERATIONS OF FAT INTAKE IN ANIMALS}

Predominant phyla documented in animal models are often Bacteroidetes and Firmicutes, and it should be noted that premature infants reveal different patterns of phyla predominance (5-7). Using adult mice, Liu et al. (8) investigated a low fat diet (19\% of TE as fat) compared with high fat diets (34-40\% of TE) with varying degrees of FA unsaturation. The high fat diet richest in saturated fatty acids (SFA) caused the largest proportional reduction, by $28 \%$, in Bacteroidetes. With higher polyunsaturated fatty acids (PUFA), smaller reductions occurred with no difference whether diets were high in $n-6$ vs. n-3 PUFA, $12 \%$ and $10 \%$ reductions, respectively. The n-3 PUFA source was flaxseed oil, containing more $\alpha$-linolenic acid (18:3n-3, LNA) than eicosapentaenoic acid (20:5n-3, EPA) or docosahexaenoic acid (22:6n-3, DHA). Turnbaugh et al. (9) also found that a diet with $40 \%$ fat, majority SFA, reduced relative abundance of Bacteroidetes relative to a lowfat diet. Phylum Firmicutes showed a relative increase with the SFA diet, including genus Enterococcus. It is noteworthy that Turnbaugh's mice were originally germ-free and had microbes obtained from human fecal specimens transplanted before dietary interventions.

Discrepancies in findings exist at lower taxonomic levels than phylum. Turnbaugh et al. (10) found increases in Mollicutes, but Hildebrandt et al. (11) showed more substantial increases in Clostridiales, both classes within Firmicutes. Both dietary interventions were high fat, predominantly SFA. These variable findings may be due to a combination of differences in the quality of fat, sources of samples (cecum vs. fecal pellets) as well as methods of sequencing and detection. The duration of dietary interventions were of considerable length but varied, ranging $3-21 \mathrm{wk}(8-10,12)$.

Different fat sources with similar degrees of saturation can impart differential changes (12). Two comparisons used high fat (38\% of TE) high SFA diets, one based on lard and one milk fat. Mice fed lard fat showed an increase in Firmicutes 
as compared with a low-fat diet ( $5 \%$ of TE), but mice fed milk fat had increased Bacteroidetes, an increase comparable with a separate group feeding a high fat PUFA-based diet. This does lend support to the notion that very specific changes in dietary FA content may have relevant influences.

PUFA effects were examined in neonatal piglets (13), one of few neonatal animal models reported on this topic. Experimental formulas were designed to mimic sow's milk and fat content was approximately $60 \%$ of TE. Fish oil (FO) or sunflower oil enrichment was added to the formula, with FO lowering the n-6:n-3 fatty acid ratio by a factor of two. PUFA content affected relative abundances but not total diversity. Proteobacteria and Actinobacteria were more prominent in the FO group. The sunflower oil group supported greater proportions of Bacteroides spp. compared with the FO group, attributable to 3 of 10 bacteria in that species.

Using mice just weaned at $4 \mathrm{wk}$ of age, Ghosh et al. (14) compared a low fat n-6 PUFA diet to two high fat diets, one with n-6 PUFA and one containing n-6 and n-3 PUFA from FO. Significantly increasing the amount of arachidonic acid (20:4n6, ARA), DHA, and EPA in the FO feeding group, there was also an increase in SFA. High fat n-6 PUFA diets showed substantial increases in relative expression of Enterobacteriaceae, segmented filamentous bacteria, and Clostridia spp. Diets containing n-3 PUFA prevented these increases and promoted growth of Bifidobacteria, Lactobacillus, and Enterococcus faecium, but not Enterococcus faecalis. Together, this model suggested that n-3 PUFA-enriched diets supported microbes considered beneficial to health and protected against growth of bacteria considered pathogenic. After dietary interventions, Citrobacter rodentium infection was induced and distal colonic histopathology assessed. The most severe damage was found with high fat n-6 PUFA diets. Although n-3 PUFA enrichment decreased colonic injury, more animals in that intervention died of sepsis after infection, highlighting potential microbial and immune system effects that may also result from dietary FA.

The mechanism of fat quality's influence on microbial composition is likely multifactorial. An indirect influence by fatinduced changes in bile acid composition has been suggested (12). A high milk-fat diet promoted growth of Bilophila wadsworthia, a change not established with a high fat PUFA-based diet. Considering that hydrophobic milk fat could change liver conjugation of bile acids, analysis of gall bladder aspirates identified a significant increase in taurine-conjugated bile acid, a rich source of sulfur for the sulfur-reducing microbe. There are likely also effects from direct exposure of intestinal microbes to FA. A high fat, high SFA palm oil diet caused increased fecal fat in mice (15). This "overflow," or decreased absorption and increased intestinal SFA transport, was associated with a decrease in diversity index as well as increased relative amounts of Firmicutes members Bacilli and Clostridia. Decreased diversity has been found by others (10). This may have relevance to the premature infant, as discussed below.

Differential effects of PUFA on growth and viability of Lactobacillus have been evaluated in vitro. Lactobacillus GG,
Lactobacillus bulgaricus and Lactobacillus casei Shirota strains were exposed to increasing concentrations of linoleic acid (18:2n-6, LA), LNA, $\gamma$-LNA, ARA, and DHA individually in growth media (16). Higher concentrations of PUFA inhibited growth, each PUFA having a different threshold concentration of impact, which also varied by strain. In surviving bacteria, viability as measured by membrane permeability was not compromised by any PUFA at any concentration. ARA and $\gamma$-LNA at low doses actually supported growth compared with controls. Exposures such as this in vivo, with stepwise changes in concentrations and assessments of species level effects, have not been reported.

\section{CHANGES IN DIETARY FAT INTAKE AND EFFECTS IN HUMANS}

Although growing literature exists regarding dietary influences on the microbiome in humans, measures of dietary fat influences are limited. Nielsen et al. (17) randomized 9-mo-old infants, born at term gestation, using a $2 \times 2$ randomization scheme to assess cow's milk vs. formula effects as well as the addition of FO at the time of starting complementary foods. Some infants were still breastfeeding in all groups and none had antibiotic exposure. Stool samples were obtained after 1 mo of intervention. Using denaturing gradient gel electrophoresis, clustering separated formula and cow's milk fed infants. Amongst cow's milk fed infants only, clustering occurred based on FO exposure. This may be explained by the baseline availability of $n-3$ PUFA in the formula.

A 9-mo-long intervention in 132 infants added PUFA, either EPA and DHA provided as FO or LA provided as sunflower oil, starting at 9 mo of age (18). Dietary intake was otherwise not prescribed and TE and macronutrient intake was reportedly similar between groups. In 15 cases of antibiotic exposure, stool samples were not collected until after $2 \mathrm{wk}$ post exposure. Bacterial diversity increased at 18 mo in the entire cohort and it appeared that Bacteroidetes became more abundant. FO exposure increased bacterial diversity only amongst infants breastfeeding at enrollment. Principal component analysis produced no groupings based on FO intervention. Significant changes in a few terminal restriction fragment frequencies occurred with FO exposure. Many findings were significantly affected by duration of breastfeeding.

Evaluating children 1-6 y of age, De Filippo et al. (19) took advantage of natural differences in dietary habits between rural Africa and Western Europe. Dietary fat constituted approximately $25-28 \%$ of TE in the African cohort and $44-47 \%$ in European children. Although the four predominant phyla were similar between these groups, Actinobacteria and Bacteroidetes were increased in the African children and Firmicutes and Proteobacteria were represented less. Bacteroidetes and Firmicutes created the most substantial distinctions between the two groups. There were other nutritional differences between these cohorts, including total duration of breastfeeding, calorie intake, protein sources, and amounts of fiber, preventing conclusions based on any specific nutrient. 


\section{Fat intake and microbiome in infants}

Awareness of findings in adult populations may help with future investigations of the role of fat in intestinal microbial alterations as comparable interventional or observational studies do not currently exist in the neonate, to the best of our knowledge. Adult subjects at risk for metabolic syndrome were assigned to a high fat (38\% of TE) high SFA diet or one of four other interventions varying total fat and ratios of unsaturation, all with equal TE (20). PUFA were consistently $6 \%$ of TE in all intervention arms and diets lasted at least $24 \mathrm{wk}$. Lower fat (28\% of TE), lower SFA diet increased Bacteroides and Bifidobacterium spp. and monounsaturated FA had no apparent influence. The high fat interventions reduced the total fecal bacteria count. Dietary recalls analyzed by $\mathrm{Wu}$ et al. (21) showed positive associations between SFA intake and Bacteroidetes as well as Actinobacteria, and negative associations with Firmicutes and Proteobacteria. In addition, transFA, to be discussed later, also showed positive and negative associations. The false discovery rate used in models was considered high at 25\%. Walker et al. (22) found changes within Firmicutes and Actinobacteria but not Bacteroidetes using a crossover design; however, within individual intervention arms, there were extremes of fat intake as \% of TE.

In a Finnish cohort of monozygotic twins, monounsaturated FA and n-6 PUFA intake inversely correlated with numbers of Bifidobacteria and n-3 PUFA intake positively correlated with numbers of Lactobacillus (23). Interestingly, similar SFA intake reduced interindividual differences, promoting similarity in Bacteroides spp. between twins. This population had a range of $\mathrm{BMI}$, allowing investigators to clarify that energy and fat intake, not BMI, were affecting microbial composition. Combining observational and interventional data, others supported that only a long-term dietary intervention could minimize interindividual variability (21).

Looking more broadly at the strength of influence of fat, in a separate Finnish cohort, total PUFA, LA, and LNA intake as $\%$ of TE each influenced the relative abundance of a cluster of Bacteroides spp., contributing as much as $30 \%$ of the variation (24). For multiple species within a Clostridium cluster, over $10 \%$ to approximately $30 \%$ of variation was related to \% of TE from PUFA and LA as well as intake of LA, LNA, and DHA in grams per day.

Advocating appropriate caution in making associations between nutritional intake and influences on microbes, Cilieborg et al. (25) cite methodological differences and a lack of accounting for nonnutritive factors in milk amongst many reasons for variable findings. Multiple nutritive and nonnutritive factors, including oligosaccharides, lactoferrin and bacteria in breast milk, affect both microbial and gut development (26). Evaluating these influences in relation to one another is key and yet may prove challenging in infants. Also, the existing microbiome prior to dietary changes exerts its own influence on susceptibility to change.

Although outcomes vary, collectively results suggest that FA balance influences gut microbial flora. Further studies are necessary to confirm potentially important changes on the intestinal microbial environment. Manipulations of FA intake in premature infants do exist in study environments (27-29) and future studies could couple to evaluate microbial changes.

\section{FAT INTAKE AND ABSORPTION IN PRETERM INFANTS: VARIABLE EXPOSURES FOR THE MICROBIOME}

Breast milk lipid content highly correlates with the TE content making it a significant factor in TE intake of breastfeeding or breast milk fed infants (30). Human milk fat concentrations display considerable interindividual variability. Explanations have included maternal dietary nitrogen intake, measures of adiposity, and resumption of menstruation, yet results are conflicting and the reasons for the variability remain to be completely understood (30,31). Total fat concentrations in milk expressed from women who deliver preterm infants may be higher than in milk from women delivering at term gestation (32). In 113 German women sampled through $8 \mathrm{wk}$ postpartum, milk fat concentrations increased with lactation duration. Analysis of 2,554 samples of donated milk produced by 244 women at a Danish milk bank revealed a large range in fat content showing concentrations of $1.8 \mathrm{~g} / \mathrm{dl}$ at the lower 2.5 percentile and $8.9 \mathrm{~g} /$ $\mathrm{dl}$ at the upper 97.5 percentile (31). In 415 nationwide samples from 273 women in the United States, an extreme range of $0.7-$ $7.06 \mathrm{~g} / \mathrm{dl}$ was seen (33). The coefficient of variation for fat was the largest of the three macronutrients at $21 \%$.

As with the total lipids, specific FA content of human milk shows variability. Breast milk FA, largely in the form of triglycerides, are supplied by maternal plasma FA, partly reflecting maternal diet, and FA synthesis in the mammary gland (34). Early interventions documenting the impact of maternal diet on breast milk FA composition housed a single subject (and her newborn) in a "metabolic ward" for 7 wk (35). Introducing meals with corn oil as the predominant fat, either $40 \%$ or $70 \%$ of TE, an approximately sixfold increase in milk LA + ALA occurred. This reflected the predominance of LA in corn oil, and the concomitant decreases in SFA and oleic acid highlight that milk FA content shifts relative to dietary intake.

Human milk DHA and ARA content is well studied given potential implications on infant development. Brenna et al. (36) evaluated DHA and ARA in milk expressed from women worldwide who delivered at term gestation. The range of DHA and ARA, as \% of total FA, was $0.06-1.40$ and $0.24-1.0$, respectively (36). Coastal populations consuming marine foods, rich in preformed DHA, have higher milk DHA concentrations (36-38). Some but not all investigations show higher concentrations of saturated and PUFA in milk of women who delivered preterm (39). With diverse dietary habits, even trans-FA are found in breast milk. Southwestern American women showed some of the highest trans-FA content in the world, approximately $7 \%$ of total FA (37). Declining levels of trans-fat content in Canadian women reflect population-based changes in dietary habits resulting from public health measures, specifically education of potential health risks and clearer food labeling of trans-FA content (40). Differences in breast milk total fat content compound variability in FA. With changing volumes fed to infants, quantities and quality of fat intake by premature infants vary significantly. 
Intestinal fat absorption may be reduced in the premature infant, increasing fat exposure to microbes in distal intestines, potentially influencing microbial diversity. Impaired lipid digestion and absorption is likely a result of decreased production of multiple factors including gastric lipase, intestinal intraluminal bile salts, and exocrine pancreatic hormones (41). Bile salt-stimulated lipase is present in human milk and shows wide specificity for any lipid products and no discrimination regarding cleavage at the sn-positions of the triglyceride, increasing the amount and speed of release of FA from the triglyceride (42).

Bile salt-stimulated lipase function is reduced by heating milk. Measuring fecal fat, a small cohort $(n=5)$ of very-lowbirth-weight infants showed decreased fat absorption rates in a crossover study, when their mother's own milk was pasteurized prior to feedings (43). Fat content appeared to be just under $4 \mathrm{~g} / \mathrm{dl}$. The mean net absorption coefficient of $88 \%$ with unpasteurized milk decreased by $17 \%$ after pasteurization, although not statistically significant likely due to sample size $(P=0.063)$. Donor human milk, with approximately $3 \mathrm{~g} /$ dl total fat content, was fed to very-low-birth-weight infants in three forms: unprocessed, pasteurized, and boiled (44). Raw milk allowed the highest absorption, 73.6\%, and a significant reduction occurred with either method of heat treatment, as low as $46 \%$ with boiled milk feedings. A subject with the highest fat intake, $9.88 \mathrm{~g} / \mathrm{kg}$ per day, showed the lowest fat absorption as a composite rate from all three milk exposures. An inverse relationship was found between weight based fat intake and percentage absorbed.

An important methodological difference amongst studies is the temperature to which milk is heated, $37^{\circ} \mathrm{C}$ in Andersson's cohort and $63^{\circ} \mathrm{C}$ in Williamson's $(43,44)$. Higher temperatures may cause a larger reduction in bile salt-stimulated lipase and thus greater impairment in fat absorption. Commercial formulas lack bile salt-stimulated lipase, a partial explanation of decreased triglyceride cleavage and fat absorption in formula fed infants $(42,45)$. Chappell et al. (46) documented reductions in total fat absorption and coefficients of absorptions for medium and long chain FA in very-low-birth-weight infants fed formula as compared with a breast milk-fed group with comparable fat intake. Although it is believed that premature infants have impaired fat absorption, rates comparable with term infants, even with formula feedings, are documented $(41,47)$. This reflects the variability in dietary interventions and methodologies assessing fat absorption. Also, many studies of fat absorption were completed prior to routine addition of longer chain PUFA to commercial formulas and coefficient of absorption increases with higher degrees of unsaturation. Thus, despite the ability to measure FA content in enteral feedings for premature infants, the amounts absorbed and/or undigested may also affect microbial diversity.

\section{NECROTIZING ENTEROCOLITIS: DYSBIOSIS AND PUFA INTERACTIONS WITH TLR4}

Varying methodologies have assessed intestinal microbial patterns in premature infants that developed NEC. A predominance of Proteobacteria (Gram negative) and a marked reduction of Firmicutes have been found in fecal samples from infants developing NEC compared with control infants (48-50). Decreased diversity was apparent in NEC $(48,49)$ yet Mai et al. $(50)$ did not find this in samples collected within the week prior to diagnosis. Morrow et al. (48) and Mai et al. (50) detected changes in Actinobacteria, with decreases at or within the phylum level, yet the phylum was not reported by others (49). Some inconsistencies may result from different colonization patterns at different institutions and differences in timing of sampling. Within a single cohort, different patterns developed in samples obtained from days 4-9 vs. 10-16 (48).

TLR4 activation triggers a signaling cascade, including NFKB activation, leading to increased gene expression and production of proinflammatory mediators (51). Intestinal epithelial TLR4 expression should decrease with postnatal development and its expression remains elevated in all gut segments in experimental models of NEC $(52,53)$. With lipopolysaccharide as the ligand for murine TLR4, increased expression coupled with Gram-negative bacteria exposure provides a plausible mechanism for development of NEC $(54,55)$. Gram-negative bacteria have been more commonly identified in neonatal rats developing NEC compared with controls (55).

The degree of FA unsaturation has affected TLR4 signaling. In murine cell cultures, SFA induced expression of downstream products of TLR4 activation in a dose-related manner (56). Mono- and polyunsaturated FA mitigated signaling activation caused by SFA. This supported regulation by FA at TLR4 receptor level but did not delineate whether the receptor directly interacted with the FA. Neonatal rats fed PUFA in an established model of NEC had lower incidences of NEC compared with control animals fed formula without PUFA (53). Different types of PUFA exposure occurred; egg phospholipid, ARA and DHA, or DHA alone were added to the control formula. Lower rates of NEC correlated with decreased TLR4 mRNA expression in all four intestinal segments in rats fed PUFA. In vitro experiments in that same study showed an inhibitory effect of PUFA on platelet activating factor receptor in ileal and colonic cells (53), providing another avenue for protection against intestinal injury.

Although not measuring affects related to TLR4, rats with reduced incidences of NEC had decreased plasma levels of lipopolysaccharide in animals supplemented with PUFA (57). In this context, independent of the influence that FA may have on the microbiome, FA impart additional effects influencing downstream proinflammatory signaling, intestinal integrity, and susceptibility to NEC.

\section{CONCLUSION}

Collection and analysis of detailed nutrient provisions to premature infants is feasible in studies evaluating the intestinal microbiome. Measuring breast milk composition allows for documentation of fat intake. Measuring metabolism (i.e., FA cleavage and absorption) is not as straight forward. Fat is a predominant energy source and lipid subcomponents are vital for growth and brain development throughout infancy and childhood, and naturally occurring variability can be used 
in cohort analyses. Conflicting data of the dietary influences on the microbiome exist, as well as uncertainty of any given microbiome's influence on disease risk, and there remains a paucity of evidence in human infants. Variable findings result from different methodologies of microbial detection and timing of sampling, and possibly from a lack of depth of accounting for specific nutrient intake. Statistical analyses measuring microbial changes specifically due to lipids is challenging considering the numerous factors in breast milk that influence the microbiome. It is possible that dietary FA cause minimal influence on microbes as related to risk of disease, but there are still likely important influences of FA on immune system function, or dysfunction, at the level of the intestinal mucosa. Growing evidence suggests value in reporting specific nutrient intake, more than just breast milk or formula feedings, in investigations measuring influences on the intestinal microbiome in premature infants.

\section{STATEMENT OF FINANCIAL SUPPORT AND DISCLOSURE}

None.

Disclosure: None.

\section{REFERENCES}

1. Saavedra JM, Dattilo AM. Early development of intestinal microbiota: implications for future health. Gastroenterol Clin North Am 2012;41:717-31.

2. Yatsunenko T, Rey FE, Manary MJ, et al. Human gut microbiome viewed across age and geography. Nature 2012;486:222-7.

3. Arboleya S, Binetti A, Salazar N, et al. Establishment and development of intestinal microbiota in preterm neonates. FEMS Microbiol Ecol 2012;79:763-72.

4. Arboleya S, Ang L, Margolles A, et al. Deep 16S rRNA metagenomics and quantitative PCR analyses of the premature infant fecal microbiota. Anaerobe 2012;18:378-80.

5. Schwiertz A, Gruhl B, Löbnitz M, Michel P, Radke M, Blaut M. Development of the intestinal bacterial composition in hospitalized preterm infants in comparison with breast-fed, full-term infants. Pediatr Res 2003;54:393-9.

6. Gewolb IH, Schwalbe RS, Taciak VL, Harrison TS, Panigrahi P. Stool microflora in extremely low birthweight infants. Arch Dis Child Fetal Neonatal Ed 1999;80:F167-73.

7. Penders J, Thijs C, Vink C, et al. Factors influencing the composition of the intestinal microbiota in early infancy. Pediatrics 2006;118:511-21.

8. Liu T, Hougen H, Vollmer AC, Hiebert SM. Gut bacteria profiles of Mus musculus at the phylum and family levels are influenced by saturation of dietary fatty acids. Anaerobe 2012;18:331-7.

9. Turnbaugh PJ, Ridaura VK, Faith JJ, Rey FE, Knight R, Gordon JI. The effect of diet on the human gut microbiome: a metagenomic analysis in humanized gnotobiotic mice. Sci Transl Med 2009;1:6ra14.

10. Turnbaugh PJ, Bäckhed F, Fulton L, Gordon JI. Diet-induced obesity is linked to marked but reversible alterations in the mouse distal gut microbiome. Cell Host Microbe 2008;3:213-23.

11. Hildebrandt MA, Hoffmann C, Sherrill-Mix SA, et al. High-fat diet determines the composition of the murine gut microbiome independently of obesity. Gastroenterology 2009;137:1716-24.e1-2.

12. Devkota S, Wang Y, Musch MW, et al. Dietary-fat-induced taurocholic acid promotes pathobiont expansion and colitis in Il10-/- mice. Nature 2012;487:104-8.

13. Andersen AD, Mølbak L, Thymann T, Michaelsen KF, Lauritzen L. Dietary long-chain n-3 PUFA, gut microbiota and fat mass in early postnatal piglet development-exploring a potential interplay. Prostaglandins Leukot Essent Fatty Acids 2011;85:345-51.

14. Ghosh S, DeCoffe D, Brown K, et al. Fish oil attenuates omega- 6 polyunsaturated fatty acid-induced dysbiosis and infectious colitis but impairs LPS dephosphorylation activity causing sepsis. PLoS One 2013;8:e55468.
15. de Wit N, Derrien M, Bosch-Vermeulen H, et al. Saturated fat stimulates obesity and hepatic steatosis and affects gut microbiota composition by an enhanced overflow of dietary fat to the distal intestine. Am J Physiol Gastrointest Liver Physiol 2012;303:G589-99.

16. Kankaanpää PE, Salminen SJ, Isolauri E, Lee YK. The influence of polyunsaturated fatty acids on probiotic growth and adhesion. FEMS Microbiol Lett 2001;194:149-53.

17. Nielsen S, Nielsen DS, Lauritzen L, Jakobsen M, Michaelsen KF. Impact of diet on the intestinal microbiota in 10-month-old infants. J Pediatr Gastroenterol Nutr 2007;44:613-8.

18. Andersen AD, Mølbak L, Michaelsen KF, Lauritzen L. Molecular fingerprints of the human fecal microbiota from 9 to 18 months old and the effect of fish oil supplementation. J Pediatr Gastroenterol Nutr 2011;53:303-9.

19. De Filippo C, Cavalieri D, Di Paola M, et al. Impact of diet in shaping gut microbiota revealed by a comparative study in children from Europe and rural Africa. Proc Natl Acad Sci USA 2010;107:14691-6.

20. Fava F, Gitau R, Griffin BA, Gibson GR, Tuohy KM, Lovegrove JA. The type and quantity of dietary fat and carbohydrate alter faecal microbiome and short-chain fatty acid excretion in a metabolic syndrome 'at-risk' population. Int J Obes (Lond) 2013;37:216-23.

21. Wu GD, Chen J, Hoffmann C, et al. Linking long-term dietary patterns with gut microbial enterotypes. Science 2011;334:105-8.

22. Walker AW, Ince J, Duncan SH, et al. Dominant and diet-responsive groups of bacteria within the human colonic microbiota. ISME J 2011;5:220-30.

23. Simões CD, Maukonen J, Kaprio J, Rissanen A, Pietiläinen KH, Saarela M. Habitual dietary intake is associated with stool microbiota composition in monozygotic twins. J Nutr 2013;143:417-23.

24. Lappi J, Salojärvi J, Kolehmainen M, et al. Intake of whole-grain and fiberrich rye bread versus refined wheat bread does not differentiate intestinal microbiota composition in Finnish adults with metabolic syndrome. J Nutr 2013;143:648-55.

25. Cilieborg MS, Boye M, Sangild PT. Bacterial colonization and gut development in preterm neonates. Early Hum Dev 2012;88:Suppl 1:S41-9.

26. Berrington JE, Stewart CJ, Embleton ND, Cummings SP. Gut microbiota in preterm infants: assessment and relevance to health and disease. Arch Dis Child Fetal Neonatal Ed 2013;98:F286-90.

27. Makrides M, Gibson RA, McPhee AJ, et al. Neurodevelopmental outcomes of preterm infants fed high-dose docosahexaenoic acid: a randomized controlled trial. JAMA 2009;301:175-82.

28. Henriksen C, Haugholt K, Lindgren M, et al. Improved cognitive development among preterm infants attributable to early supplementation of human milk with docosahexaenoic acid and arachidonic acid. Pediatrics 2008;121:1137-45.

29. Carlson SE, Werkman SH, Rhodes PG, Tolley EA. Visual-acuity development in healthy preterm infants: effect of marine-oil supplementation. Am J Clin Nutr 1993;58:35-42.

30. Nommsen LA, Lovelady CA, Heinig MJ, Lönnerdal B, Dewey KG. Determinants of energy, protein, lipid, and lactose concentrations in human milk during the first 12 mo of lactation: the DARLING Study. Am J Clin Nutr 1991;53:457-65.

31. Michaelsen KF, Skafte L, Badsberg JH, Jørgensen M. Variation in macronutrients in human bank milk: influencing factors and implications for human milk banking. J Pediatr Gastroenterol Nutr 1990;11:229-39.

32. Bauer J, Gerss J. Longitudinal analysis of macronutrients and minerals in human milk produced by mothers of preterm infants. Clin Nutr 2011;30:215-20.

33. Wojcik KY, Rechtman DJ, Lee ML, Montoya A, Medo ET. Macronutrient analysis of a nationwide sample of donor breast milk. J Am Diet Assoc 2009;109:137-40.

34. German JB, Dillard CJ. Composition, structure and absorption of milk lipids: a source of energy, fat-soluble nutrients and bioactive molecules. Crit Rev Food Sci Nutr 2006;46:57-92.

35. Insull W Jr, Hirsch J, James T, Ahrens EH Jr. The fatty acids of human milk. II. Alterations produced by manipulation of caloric balance and exchange of dietary fats. J Clin Invest 1959;38:443-50. 
36. Brenna JT, Varamini B, Jensen RG, Diersen-Schade DA, Boettcher JA, Arterburn LM. Docosahexaenoic and arachidonic acid concentrations in human breast milk worldwide. Am J Clin Nutr 2007;85:1457-64.

37. Mosley EE, Wright AL, McGuire MK, McGuire MA. trans Fatty acids in milk produced by women in the United States. Am J Clin Nutr 2005;82:1292-7.

38. Wang L, Shimizu Y, Kaneko S, et al. Comparison of the fatty acid composition of total lipids and phospholipids in breast milk from Japanese women. Pediatr Int 2000;42:14-20.

39. Bokor S, Koletzko B, Decsi T. Systematic review of fatty acid composition of human milk from mothers of preterm compared to full-term infants. Ann Nutr Metab 2007;51:550-6.

40. Friesen R, Innis SM. Trans fatty acids in human milk in Canada declined with the introduction of trans fat food labeling. J Nutr 2006;136:2558-61.

41. Lindquist $\mathrm{S}$, Hernell O. Lipid digestion and absorption in early life: an update. Curr Opin Clin Nutr Metab Care 2010;13:314-20.

42. Hernell O, Bläckberg L, Chen Q, Sternby B, Nilsson A. Does the bile salt-stimulated lipase of human milk have a role in the use of the milk long-chain polyunsaturated fatty acids? J Pediatr Gastroenterol Nutr 1993;16:426-31.

43. Andersson Y, Sävman K, Bläckberg L, Hernell O. Pasteurization of mother's own milk reduces fat absorption and growth in preterm infants. Acta Paediatr 2007;96:1445-9.

44. Williamson S, Finucane E, Ellis H, Gamsu HR. Effect of heat treatment of human milk on absorption of nitrogen, fat, sodium, calcium, and phosphorus by preterm infants. Arch Dis Child 1978;53:555-63.

45. Carnielli VP, Verlato G, Pederzini F, et al. Intestinal absorption of longchain polyunsaturated fatty acids in preterm infants fed breast milk or formula. Am J Clin Nutr 1998;67:97-103.

46. Chappell JE, Clandinin MT, Kearney-Volpe C, Reichman B, Swyer PW. Fatty acid balance studies in premature infants fed human milk or formula: effect of calcium supplementation. J Pediatr 1986;108:439-47.
47. Rings EH, Minich DM, Vonk RJ, Stellaard F, Fetter WP, Verkade HJ. Functional development of fat absorption in term and preterm neonates strongly correlates with ability to absorb long-chain fatty acids from intestinal lumen. Pediatr Res 2002;51:57-63.

48. Morrow AL, Lagomarcino AJ, Schibler KR, et al. Early microbial and metabolomic signatures predict later onset of necrotizing enterocolitis in preterm infants. Microbiome 2013;1:13.

49. Wang Y, Hoenig JD, Malin KJ, et al. 16S rRNA gene-based analysis of fecal microbiota from preterm infants with and without necrotizing enterocolitis. ISME J 2009;3:944-54.

50. Mai V, Young CM, Ukhanova M, et al. Fecal microbiota in premature infants prior to necrotizing enterocolitis. PLoS One 2011;6:e20647.

51. Akira S, Takeda K. Toll-like receptor signalling. Nat Rev Immunol 2004;4:499-511.

52. Jilling T, Simon D, Lu J, et al. The roles of bacteria and TLR4 in rat and murine models of necrotizing enterocolitis. J Immunol 2006;177:3273-82.

53. Lu J, Jilling T, Li D, Caplan MS. Polyunsaturated fatty acid supplementation alters proinflammatory gene expression and reduces the incidence of necrotizing enterocolitis in a neonatal rat model. Pediatr Res 2007;61:427-32.

54. Chan KL, Ho JC, Chan KW, Tam PK. A study of gut immunity to enteral endotoxin in rats of different ages: a possible cause for necrotizing enterocolitis. J Pediatr Surg 2002;37:1435-40.

55. Carlisle EM, Poroyko V, Caplan MS, Alverdy JA, Liu D. Gram negative bacteria are associated with the early stages of necrotizing enterocolitis. PLoS One 2011;6:e18084.

56. Lee JY, Sohn KH, Rhee SH, Hwang D. Saturated fatty acids, but not unsaturated fatty acids, induce the expression of cyclooxygenase- 2 mediated through toll-like receptor 4. J Biol Chem 2001;276:16683-9.

57. Caplan MS, Russell T, Xiao Y, Amer M, Kaup S, Jilling T. Effect of polyunsaturated fatty acid (PUFA) supplementation on intestinal inflammation and necrotizing enterocolitis (NEC) in a neonatal rat model. Pediatr Res 2001;49:647-52. 